\title{
Experimental Modal Analysis - A Tool for Unbalance Identification of Rotating Machines*
}

\author{
T. KREUZINGER-JANIK ${ }^{\dagger}$ and H. IRRETIER \\ Institute of Mechanics, University of Kassel, Moenchebergstr. 7, 34109 Kassel, Germany
}

(Received 12 May 1998; In final form 17 July 1998)

\begin{abstract}
In this paper a method is proposed for unbalance identification of elastic rotors. The method is essentially based on the rotordynamic theory combined with experimental modal analysis and allows to identify the unbalance distribution on the complete rotor. A rotor test rig designed for rotordynamic experiments, modal analysis and especially for the unbalance identification has been developed. It allows an arbitrary excitation with a particularly developed noncontact magnetic exciter, as well as measuring vibrations in radial direction with non-contact laser sensors and eddy currents. Special effects of rotordynamic like anisotropic journal bearings and gyroscopic forces can be simulated. Experimental and theoretical results like mode shapes and unbalance parameters for the laboratory model are presented in detail.
\end{abstract}

Keywords: Unbalance, Modal analysis, Rotordynamic, Balancing, Identification

\section{INTRODUCTION}

To balance flexible rotors, two basic techniques are currently used: modal balancing and balancing with influence coefficients (Goodman, 1964; Kellenberger, 1987). These methods have been improved in practice and numerous combinations and variations of these two basic techniques have been developed. The disadvantage of balancing rotors in this way, is the limited angular speed range, for which the rotor is finally balanced.

The proposed method allows basically the identification of the unbalance distribution on the complete rotor. For that purpose the unbalance vibration $\underline{\hat{\bar{W}}}^{\mathrm{U}}\left(\Omega_{\mathrm{R}}\right)$ must be measured at constant angular speeds $\Omega_{\mathrm{R}}$. The measurement planes may be at any location on the rotor, for example at the balancing planes or other well accessible locations. Therefore the length of the vector $\hat{\bar{W}}^{\mathrm{U}}\left(\Omega_{\mathrm{R}}\right)$ is limited by the number of measurement planes. To describe the motion of the rotor, it is necessary to measure the displacement on one measurement plane in two directions, preferably $90^{\circ}$ apart. Herewith the length of the vector $\underline{\hat{W}}^{\mathrm{U}}\left(\Omega_{\mathrm{R}}\right)$ amounts to the number of measurement points $n_{\mathrm{M}}$.

An experimental modal analysis of the rotating machines at the same angular speed $\Omega_{\mathrm{R}}$ follows. The resulting frequency response function matrix

\footnotetext{
* This paper was originally presented at ISROMAC-7.

${ }^{\dagger}$ Corresponding author. Fax: +49 (0) 561/8042720. E-mail: kreuz@ ifm.maschinenbau.uni-kassel.de.
} 
(FRFM) $\overline{\mathbf{H}}$ describes the relationship between the forced vibration - for unbalance the unbalance vibration $\underline{\hat{\bar{W}}}^{\mathrm{U}}\left(\Omega_{\mathrm{R}}\right)$ - and the exciting force - in this case the unbalance force $\underline{\bar{F}}^{\mathrm{U}}\left(\Omega_{\mathrm{R}}\right)$ :

$$
\underline{\hat{\bar{W}}}^{\mathrm{U}}\left(\Omega_{\mathrm{R}}\right)=\overline{\mathbf{H}} \cdot \underline{\overline{\hat{F}}}^{\mathrm{U}}\left(\Omega_{\mathrm{R}}\right) .
$$

Therefore, a pseudo inversion ${ }^{1}$ of the generally incomplete FRFM $\overline{\mathbf{H}}^{+}$leads to the unbalance force vector and thus to the unbalance parameters combined in the vector $\underline{\bar{\varepsilon}}(\overline{\boldsymbol{\Theta}}$ including mass, geometry and transformation parameters) by

$$
\underline{\bar{\varepsilon}}=\frac{1}{\Omega_{\mathrm{R}}^{2}} \cdot \bar{\Theta}^{+} \cdot \underline{\bar{F}}^{\mathrm{U}}\left(\Omega_{\mathrm{R}}\right) .
$$

Finally, it may be possible to identify the origin of the unbalance forces in opposite to the common balancing methods. The knowledge of the unbalance distribution of the rotor enables:

- balancing for arbitrary speed ranges (depending on the numbers of balancing planes);

- on-line monitoring of the unbalance state.

\section{DISCRETIZATION AND MODAL DESCRIPTION OF ROTATING MACHINES}

A common procedure to get a linear, discrete mechanical rotor-model is the finite-elementmethod (Krämer, 1984; Lee, 1993). Such an FEmodel consists of:

- TIMOSHENKO-beam elements to describe the elastic shaft of the rotor;

- rigid mass-elements composed of masses and inertia moments to describe discs like turbine bladed discs;

- spring- and damper-elements to describe the bearings of the rotor (in this case journal bearings).

Using the principle of virtual work, the equation of motion can be calculated as

$$
\mathbf{M} \underline{\tilde{\omega}}+(\mathbf{D}+\mathbf{G}) \underline{\dot{\bar{\omega}}}+\mathbf{K} \underline{\bar{\omega}}=\bar{f}(t) .
$$

By that the generalized displacements are combined in the complex extended vector

$$
\underline{\omega}=\operatorname{Re}\{\underline{\bar{\omega}}\} ; \quad \underline{\bar{\omega}}=\underline{\hat{\bar{W}}} \cdot \mathrm{e}^{\mathrm{j} \Omega_{\mathrm{F}} t} \quad \underline{\hat{\bar{W}}}_{i}=\underline{\hat{W}}_{i} \cdot \mathrm{e}^{\mathrm{j} \alpha_{\mathrm{W}_{i}}} \cdot
$$

This vector describes the motion of the midpoint $W_{e}$ of a beam element $e$ in the local non-rotating coordinates $x, y, z$; its dimension is $\left(n_{\mathrm{F}} \times 1\right)$ (Fig. 1). The static and dynamic properties of the system are described be the system matrices $\mathbf{M}, \mathbf{D}, \mathbf{G}, \mathbf{K}$. The mass matrix $\mathbf{M}$ is a symmetric matrix composed of the masses and inertia moments of the beam elements and discs. The gyroscopic effects of the cross section of the beam elements and the rigid discs are considered by the gyroscopic matrix $\mathbf{G}$. This matrix depends linearly on the angular speed $\Omega_{\mathrm{R}}$ of the rotor and is skew-symmetric. The properties of the journal bearings are described by the speed dependent bearing coefficients combined with the unsymmetric stiffness and damping matrices $\mathbf{K}^{\mathrm{L}}\left(\Omega_{\mathrm{R}}\right)$ and $\mathbf{D}=\mathbf{D}^{\mathrm{L}}\left(\Omega_{\mathrm{R}}\right)$. Additionally, the stiffness of the beam elements described by the symmetric stiffness matrix $\mathbf{K}^{\mathbf{B}}$, leads to the total stiffness matrix $\mathbf{K}=\mathbf{K}^{\mathrm{L}}\left(\Omega_{\mathrm{R}}\right)+\mathbf{K}^{\mathrm{B}}$.

Finally, the time dependent external forces have to be introduced by the vector $f(t)$. In general, these forces can be gearforces, steamforces, etc. In this paper only harmonic unbalance forces or magnetic exciter forces are necessary for the further computation:

$$
f(t)=\operatorname{Re}\{\underline{f}\} ; \quad \bar{f}=\underline{\hat{F}} \cdot \mathrm{e}^{\mathrm{j} \Omega_{\mathrm{F}} t} \quad \hat{\bar{F}}_{i}=\hat{F}_{i} \cdot \mathrm{e}^{\mathrm{j} \alpha_{\mathrm{F}_{i}}} .
$$

In the special case of unbalance excitation the exciting frequency $\Omega_{\mathrm{F}}$ is equal to the rotational

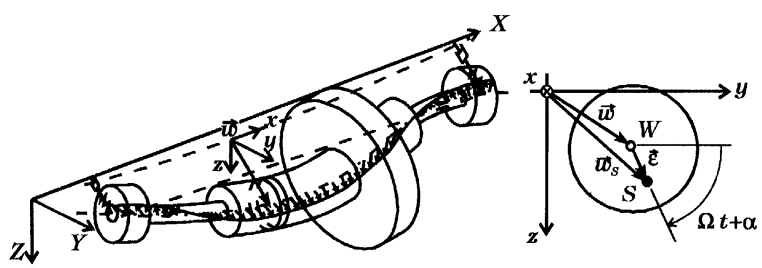

FIGURE 1 An elastic rotor-bearing model.

\footnotetext{
${ }^{1}$ Pseudo inversion $={ }^{+}$.
} 
frequency $\Omega_{\mathrm{R}}$. After a modal transformation the amplitudes of the system response can be determined by introducing the FRFM $\overline{\mathbf{H}}\left(\Omega_{\mathrm{F}}\right),{ }^{2}$ consisting of the outer product of the right- and left-hand eigenvectors $\bar{\phi}_{k}^{\mathrm{R}}, \bar{\phi}_{k}^{\mathrm{LT}}$ and the eigenvalues $\bar{\lambda}_{k}$ :

$$
\underline{\underline{W}}\left(\Omega_{\mathrm{F}}\right)=\underbrace{\left(\sum_{k=1}^{2 n_{\mathrm{F}}} \frac{\bar{\phi}_{k}^{\mathrm{R}} \bar{\phi}_{k}^{\mathrm{LT}}}{\bar{\alpha}_{k}\left(j \Omega_{\mathrm{F}}-\bar{\lambda}_{k}\right)}\right)}_{\overline{\mathbf{H}}\left(\Omega_{\mathrm{F}}\right)} \cdot \bar{F}\left(\Omega_{\mathrm{F}}\right) .
$$

The eigenvectors can be normalized by using the coefficients $\bar{\alpha}_{k}$. Regarding only harmonic excitation, Eq. (6) can be reduced to

$$
\underline{\hat{W}}=\overline{\mathbf{H}}\left(\Omega_{\mathrm{F}}\right) \cdot \underline{\hat{\bar{F}}},
$$

where the time-independent amplitude vectors $\underline{\hat{W}}$ and $\hat{\bar{F}}$ are defined in Eqs. (4) and (5).

\section{UNBALANCE PARAMETERS}

The unbalance $\bar{U}$ of the discretized rotor is a superposition of the unbalance of the beam elements $\underline{\bar{U}}^{\mathrm{B}}$ and the disc elements $\underline{\bar{U}}^{\mathrm{S}}$ :

$$
\underline{\bar{U}}=\underline{\bar{U}}^{\mathrm{B}}+\underline{\bar{U}}^{\mathrm{S}} .
$$

\section{Unbalance of the Beam Elements}

The connection curve of the centre of gravity of the beam element $e$ (Fig. 1) can be approximated by a linear eccentricity function $\varepsilon_{e}^{\mathrm{B}}(x, t)$ in $y$ - and $z$ direction. The centrifugal loading $f_{\rho}(x, t)$ of the beam elements, caused by the eccentricity $\underline{\varepsilon}_{e}^{\mathrm{B}}(x, t)$, the angular speed $\Omega_{\mathrm{R}}$ and the mass density multiplied by the cross section of the beam element $\varrho_{\mathrm{e}} \cdot A_{e}$ reads

$$
f_{-e}(x, t)=\Omega_{\mathrm{R}}^{2} \cdot \varrho_{e} \cdot A_{e} \cdot \underline{\varepsilon}_{e}^{\mathrm{B}}(x, t) .
$$

Considering the principle of the virtual work, that the virtual work of the distributed forces must be equal to the virtual work of the nodal forces i.e $\delta A^{\mathrm{distr}}=\delta A^{\text {nodal }}$ leads to the nodal centrifugal force vector

$$
f_{-e}^{\mathrm{B}}=\left[\begin{array}{c}
f_{y} \\
f_{z} \\
m_{y} \\
m_{z}
\end{array}\right]_{e}^{\mathrm{B}}=\Omega_{\mathrm{R}}^{2} \underbrace{\varrho_{e} A_{e} \int_{0}^{L_{e}} \mathbf{H}^{\mathrm{T}} \cdot \underline{\varepsilon}_{e}^{\mathrm{B}}(x, t) \mathrm{d} x}_{\underline{U}_{e}^{\mathrm{B}}},
$$

with $\mathbf{H}^{\mathrm{T}}$ as a matrix including the HERMITE polynomials. The complex extension, the calculation of the integral in Eq. (10) and the transformation into global co-ordinates, determines the global unbalance vector $\underline{\bar{U}}^{\mathrm{B}}$ of the beam elements:

$$
\underline{\bar{U}}^{\mathrm{B}}=\underbrace{\overline{\boldsymbol{\Gamma}}^{\mathrm{B}} \cdot \mathbf{M}^{\mathrm{B}}}_{\overline{\boldsymbol{\Theta}}^{\mathrm{B}}} \cdot \underline{\bar{\varepsilon}}^{\mathrm{B}} .
$$

The matrix $\overline{\boldsymbol{\Gamma}}^{\mathrm{B}}$ consists of the HERMITE polynomials, the geometry and the constitutive equations of the beam theory. The matrix $\mathbf{M}^{\mathrm{B}}$ consists the masses of the elements $\varrho_{e} A_{e} L_{e}$. The unbalance parameters are combined in the vector $\underline{\bar{\varepsilon}}^{\mathrm{B}}=\left[\cdots \varepsilon_{i}^{\mathrm{B}} \cdot \mathrm{e}^{j \alpha_{i}^{\mathrm{B}}} \cdots\right]^{\mathrm{T}}$ for each degree of freedom $i=1, \ldots, 4 n_{\mathrm{K}}$ of the model.

\section{Unbalance of the Disc Elements}

The unbalance of the disc elements are partitioned in a static and dynamic unbalance. The static unbalance force can be determined using the second Newton's law and the dynamic unbalance is calculated by the moment of momentum principle. Therefore, the complex extended vector of unbalance forces for one disc element $e=1, \ldots, n_{\mathrm{S}}$ is determined by $\left(\Delta \theta_{e}=\theta_{a e}-\theta_{p e}\right)$

$$
\bar{f}_{-e}^{\mathrm{S}}=\Omega_{\mathrm{R}}^{2} \cdot \mathrm{e}^{\mathrm{j} \Omega_{\mathrm{R}} t} \cdot \underbrace{\left[\begin{array}{cc}
m_{e}^{\mathrm{S}} & 0 \\
-j \cdot m_{e}^{\mathrm{S}} & 0 \\
0 & \Delta \theta_{e} \\
0 & -j \cdot \Delta \theta_{e}
\end{array}\right] \cdot\left[\begin{array}{c}
\bar{\varepsilon}_{e}^{\mathrm{S}} \\
\bar{\beta}_{e}^{\mathrm{S}}
\end{array}\right]}_{\underline{\tilde{U}}_{e}^{\mathrm{S}}} .
$$

\footnotetext{
${ }^{2}$ Complex conjugate $=*$.
} 
Transformation into global co-ordinates leads to the unbalance of the disc elements.

$$
\underline{\bar{U}}^{\mathrm{S}}=\overline{\boldsymbol{\Theta}}^{\mathrm{S}} \cdot \underline{\bar{\varepsilon}}^{\mathrm{S}} .
$$

\section{UNBALANCE IDENTIFICATION}

The unbalance force vector is the product of the angular speed of the rotor and the unbalance vector

$$
\bar{f}=\underline{\hat{\bar{F}}} \cdot \mathrm{e}^{j \Omega_{\mathrm{R}} t}=\Omega_{\mathrm{R}}^{2} \cdot \underline{\bar{U}} \cdot \mathrm{e}^{\mathrm{j} \Omega_{\mathrm{R}} t} .
$$

Using Eqs. (8), (11), (13) and (14) allows an estimation of the possibility to identify the unbalance parameters $\bar{\varepsilon}_{i}$ using the amplitude of the unbalance force vector $\underline{\bar{F}}$

$$
\underline{\hat{F}}=\Omega_{\mathrm{R}}^{2} \cdot \overline{\boldsymbol{\Theta}} \cdot \underline{\bar{\varepsilon}},
$$

with

$$
\overline{\boldsymbol{\Theta}}=\left[\overline{\boldsymbol{\Theta}}^{\mathrm{B}} \overline{\boldsymbol{\Theta}}^{\mathrm{S}}\right] ; \quad \overline{\bar{\varepsilon}}=\left[\begin{array}{c}
\overline{\bar{\varepsilon}}^{\mathrm{B}} \\
\underline{\underline{\varepsilon}}^{\mathrm{S}}
\end{array}\right] .
$$

Regarding the linear dependence of the $z$-components from the $y$-components of the forces and moments

$$
\hat{\bar{F}}_{y}=-j \cdot \hat{\bar{F}}_{z} ; \quad \hat{\bar{M}}_{y}=-j \cdot \hat{\bar{M}}_{z},
$$

$2 n_{\mathrm{K}}$ equations are available from Eq. (15) for calculating $\left(n_{\mathrm{K}}+2 n_{\mathrm{S}}\right)$ unknown complex unbalance parameters. In practical case $n_{\mathrm{K}}>2 n_{\mathrm{S}}$; for example a turbomachine has only 2 or 3 discs, but more than 6 measurement locations. Hence, an overdetermined equation system may be assumed, which can be solved by least squares minimization, minimizing measurement errors

$$
\underline{\bar{\varepsilon}}=\overline{\boldsymbol{\Theta}}^{+} \underline{\bar{U}},
$$

with

$$
\overline{\boldsymbol{\Theta}}^{+}=\left(\overline{\boldsymbol{\Theta}}^{\mathrm{T} *} \mathbf{W} \overline{\boldsymbol{\Theta}}\right)^{-1} \overline{\boldsymbol{\Theta}}^{\mathrm{T} *} \mathbf{W}
$$

and $\mathbf{W}$ as a weighting matrix.
At this point two basic equations are derived. In Eq. (7) the relationship between the amplitude of the harmonic exciting force $\underline{\hat{F}}$ and the forced vibration $\underline{\hat{W}}$ is given by the FRFM $\overline{\mathbf{H}}\left(\Omega_{\mathrm{F}}\right)$. In Eq. (18) the unbalance parameters $\underline{\bar{\varepsilon}}$ are determined in dependence of the unbalance force $\underline{\hat{F}}$. Therefore, by inversion of the FRFM and elimination of the force vector, the calculation of $\underline{\bar{\varepsilon}}$ by measuring the unbalance response $\underline{\hat{W}}$ of $\underline{\bar{\varepsilon}}$ is generally possible. However, because of the dimensions of the vectors and matrices, $\overline{\mathbf{H}}$ is generally not complete and of full size, and it could be singular. The reason for this fact is that there is no way to measure all degrees of freedom $4 n_{\mathrm{K}}$ of the model and to identify all $4 n_{\mathrm{K}}$ modes of the rotor.

One possibility to invert the singular FRFM presented in (Belz, 1997), is based on the assumption for bimodal balancing (Kellenberger, 1987). The theory leads to a pseudo-inversion of the FRFM in the state space which finally delivers the unknown unbalance vector

$$
\underline{\underline{U}}=\frac{1}{\Omega_{\mathrm{R}}^{2}} \cdot\left[\underline{1}^{\mathrm{T}} \underline{0}^{\mathrm{T}}\right] \cdot \overline{\boldsymbol{\Phi}}^{\mathrm{LT}+} \cdot \overline{\boldsymbol{\Lambda}} \cdot \overline{\boldsymbol{\Phi}}^{\mathrm{R}+} \cdot\left[\begin{array}{c}
\underline{\hat{\bar{W}}} \\
j \Omega_{\mathrm{R}} \cdot \underline{\hat{W}}
\end{array}\right],
$$

with the spectral matrix

$$
\overline{\boldsymbol{\Lambda}}=\operatorname{diag}\left\{\bar{\alpha}_{k}\left(j \Omega_{\mathrm{R}}-\bar{\lambda}_{k}\right)\right\}
$$

and $\bar{\Phi}^{\mathrm{R}}$ and $\bar{\Phi}^{\mathrm{L}}$ as right-hand and left-hand modal matrices, respectively.

\section{EXPERIMENTAL MODAL ANALYSIS AND UNBALANCE IDENTIFICATION OF A ROTOR TEST STAND}

\section{Rotor Test Stand Set-Up}

The rotor test stand shown in Fig. 2 is built to verify the unbalance identification algorithm and to simulate typical and special effects of real rotors. The test rotor is driven by a drive controlled (1) 


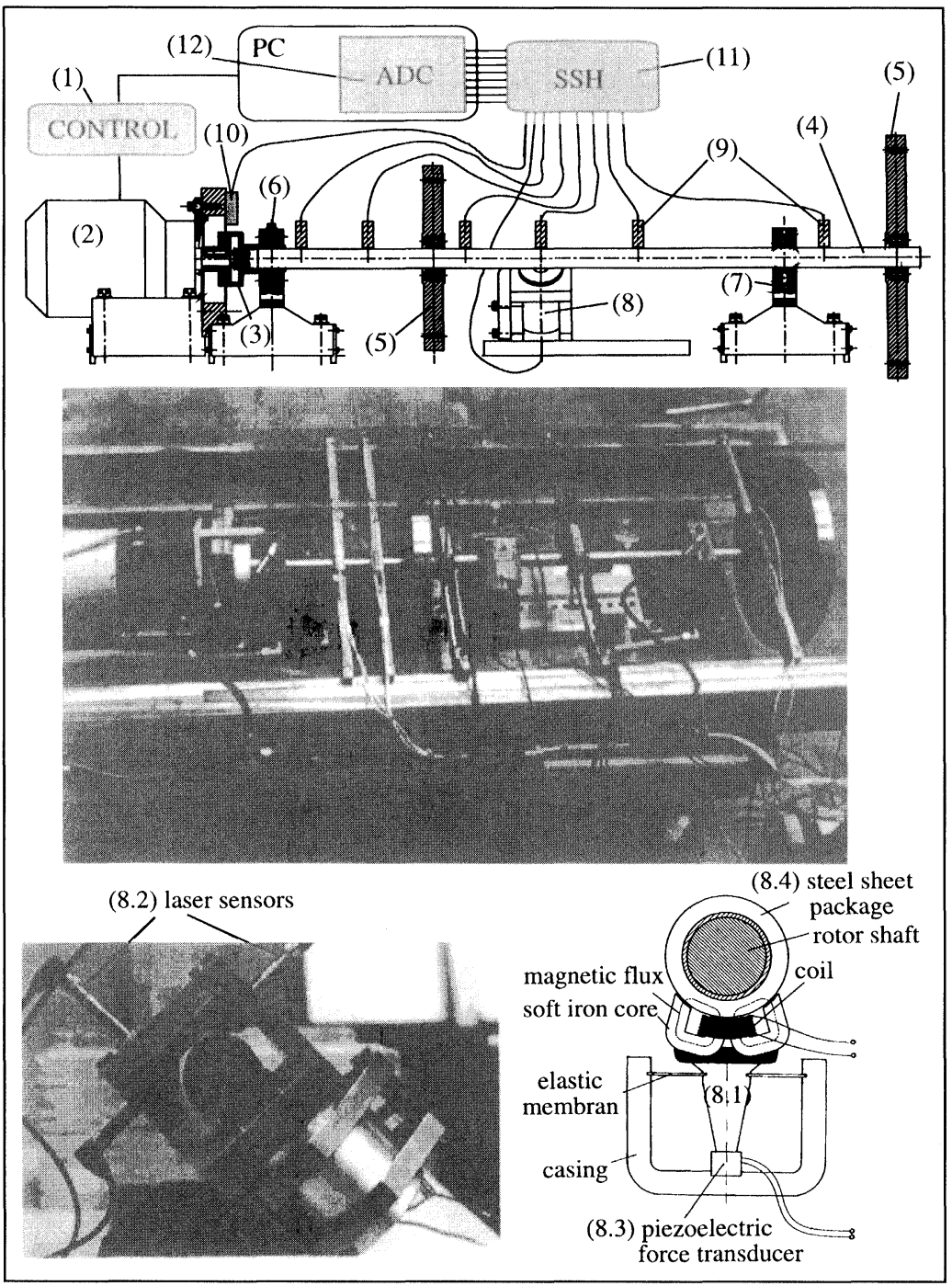

FIGURE 2 The rotor test stand.

asynchronous motor (ASM) (2), which is coupled to the shaft by a non-contact magnetic coupling (3) for no disturbance by the ASM.

On the surface tempered shaft (4), two discs (5) and one coupling (3) are fixed. A special fixation of the discs allows any location on the shaft. To set test unbalances, 24 circumferential holes in each disc exist. The rotor is supported in a fixed (6) and variable (7) industrial circular journal bearing.

\section{Measurement Set-Up}

The non-contact exciter-system (8) consists of a self-developed magnetic exciter (8.1) and - to realize the driving-point measurement - two integrated laser sensors (8.2) and a piezo-force transducer (8.3) for a direct measurement of the exciting force. ${ }^{3}$ For minimizing the eddy current dissipation a steel sheet package (8.4) is fixed at the excitation

\footnotetext{
${ }^{3}$ The magnetic exciter's first eigenfrequency is much higher than the highest excitation frequency
} 
point on the shaft. The magnetic exciter is driven by a harmonic current, which originates a double frequency harmonic magnetic force on the rotor.

The displacements are detected in six free eligible measurement planes (9), by 12 non-contact displacement sensors, consisting of two orthogonal sensors for each plane. In the neighbourhood of the magnetic exciter laser sensors and in a great distance to the magnetic exciter eddy current sensors are used. ${ }^{4}$

Finally, the speed of the rotor and the phase information is fixed by using an infrared (IR) sensor (10), which recognizes black-white contrast. The IR-sensor produces a TTL-rectangular signal, which can be used as a reference of the rotor.

To minimize time shifting, which is caused by the multiplexer of the analog-digital-converter, a special SSH-card ${ }^{5}$ (11) for PC-application has been developed. This SSH-card collects all data simultaneously and holds them for a short time, to convert them multiplexed by the analog-digitalconverter (12).

\section{Experimental Unbalance Identification}

Starting with a small revolution $n_{0} \approx 85 \mathrm{rpm}$ the surface of the rotor shaft has been scanned. At a higher constant rotor revolution $n_{1} \gg n_{0}$, the sensors measure a superposition of the surface and the unbalance vibration. The compensation of the surface offset and fitting a harmonic function, leads to the amplitudes and phase angles of the unbalance vibrations $\underline{\hat{W}}^{\mathrm{U}}, \underline{\alpha}_{\mathrm{W}}$ for all $n_{\mathrm{M}}$ displacement sensors.

After saving the data of the unbalance vibration the non-parametric identification of the FRFM for the experimental modal analysis on the operating rotor can be started. For that purpose, several exciter techniques are available, which are mainly distinguished by the test signals. Preferably for this application step sine testing or step periodic sweep testing should be used, as in the higher frequency range the force amplitude decays exponentially. The reason for this effect can be explained by the eddy current dissipations, which are minimized by the steel sheet package, but which are not eliminated for higher frequencies. To get the highest exciter energy into the rotor shaft, the step sine testing is used with equidistant frequency steps in several frequency ranges of the eigenfrequencies.

Exciting the rotor at one location and measuring the answer at the constant speed $n_{1}$ for all measuring locations $n_{\mathrm{M}}$, the variation of the measurement points can be finished. Remembering the unsymmetry of the system matrices, the FRFM is unsymmetric, too. Thus, a variation of the exciter location is necessary to measure one row and one column of the FRFM.

For the parametric identification, the modal parameters are determined by a modified orthogonal polynomial (MOP) algorithm, described in detail by Ebersbach (1989).

Using the determined modal parameters $\overline{\boldsymbol{\phi}}^{\mathrm{R}}, \overline{\boldsymbol{\phi}}^{\mathrm{L}}, \overline{\boldsymbol{\Lambda}}$ and the measured unbalance response $\underline{\hat{W}}^{\mathrm{U}}$, the unbalance vector $\underline{\bar{U}}$ can be calculated at the constant rotor speed $\Omega_{\mathrm{R}}$ by Eq. (20).

\section{THEORETICAL AND EXPERIMENTAL RESULTS}

\section{The First Rotor Test Stand Generation}

The first experiments were realized on a rotor test stand consisting of smaller discs and an old magnetic exciter (Kreuzinger-Janik, 1995). This old magnetic exciter was developed to excite plane structures. Therefore and by exciting the rotor shaft without a steel sheet package, only the first mode shape could be identified (Fig. 3). For the same rotor in (Belz, 1997) results are presented for the unbalance parameter identification, by using simulated data.

\footnotetext{
${ }^{4}$ The eddy current sensors do not work in the magnetic field, because the permeability of steel changes in dependence of the magnetic field.

${ }^{5} \mathrm{SSH}=$ Simultaneous sample and hold.
} 


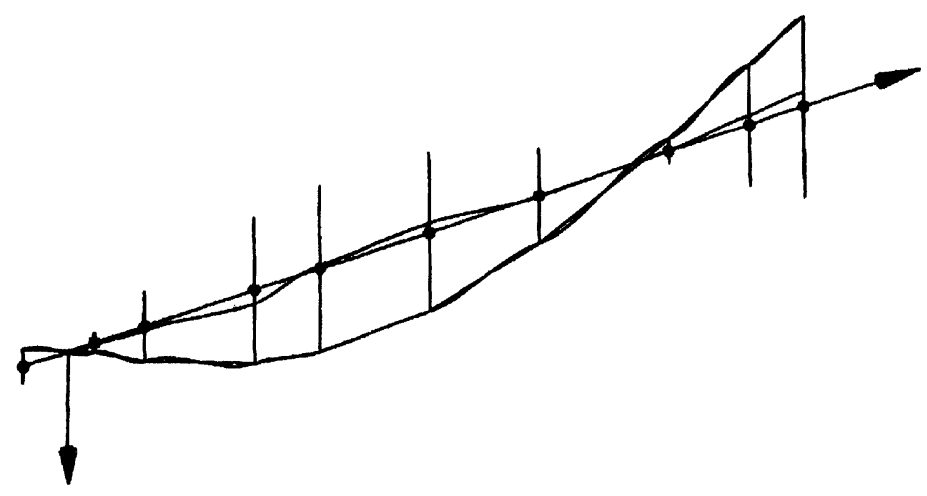

FIGURE 3 First bending mode of the rotor test stand (first generation) at $700 \mathrm{rpm}$ (Kreuzinger-Janik, 1995).

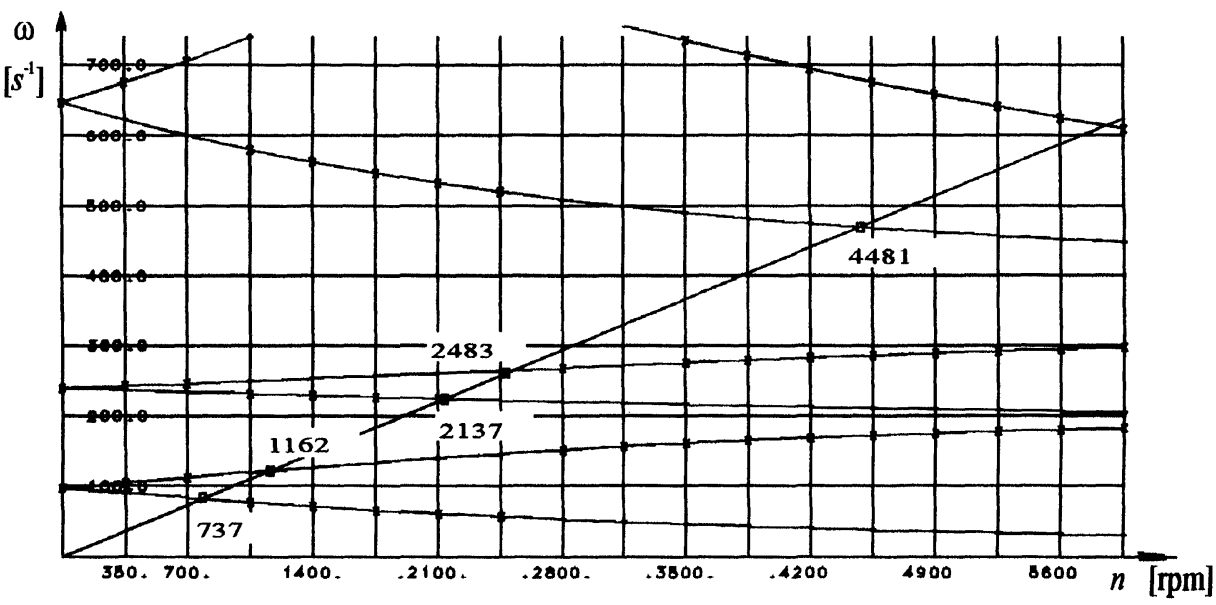

FIGURE 4 A calculated CAMPBELL-diagram

\section{The Second Rotor Test Stand Generation}

The second rotor test stand generation, described in Section 5 , has been optimized in the disc constellation by using much larger discs, optimized laser sensors and a new developed magnetic exciter system especially for rotor excitation.

The calculated CAMPBELL-diagram is shown in Fig. 4. By using a step periodic sweep, a frequency response function (FRF) at the driving point at $700 \mathrm{rpm}$ could be measured (Fig. 5). It is visible that both the forward as well as the backward whirl are excitable by this magnetic exciter.

\section{CONCLUSIONS}

In this paper the experimental modal analysis is presented to identify the unbalance distribution of rotors. After preparing the description of the unbalance parameters, the unbalance identification based on the bimodal balancing theory is outlined. A rotor test rig to verify the unbalance identification theory was described and finally, first theoretical and experimental results are presented.

Actually the experimental modal analysis is used to calculate the FRFM, for all measurement points $n_{\mathrm{M}}$. Finally, by using such FRFs shown in Fig. 5, the unbalance identification of the presented test rotor will be realized in near future. 


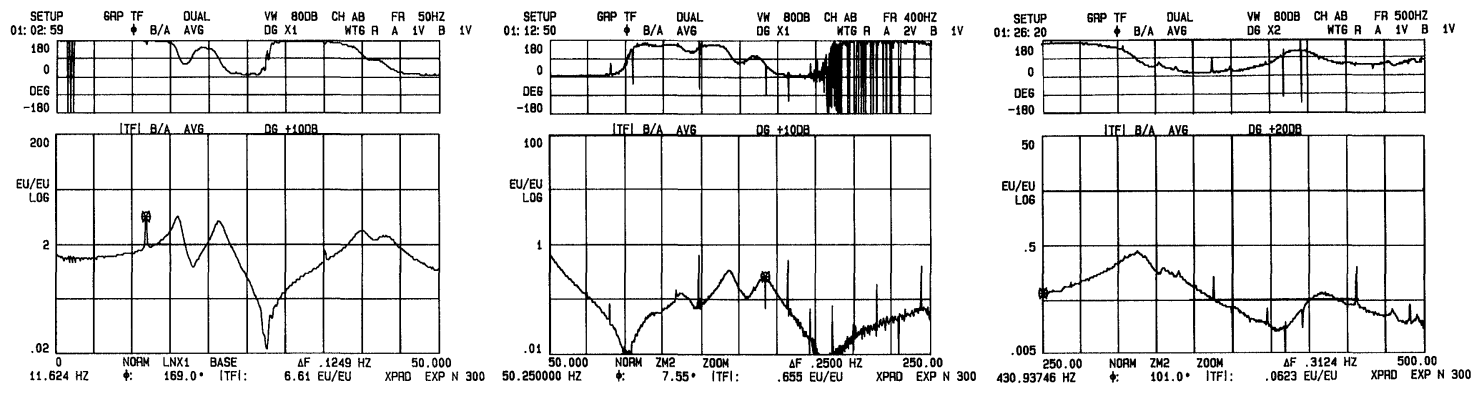

FIGURE 5 Frequency response function (FRF) at the driving point $700 \mathrm{rpm}$.

\section{NOMENCLATURE}

$\begin{array}{ll}n_{\mathrm{M}} & \text { number of measurement points } \\ n_{\mathrm{F}} & \text { number of degrees of freedom } \\ \Omega_{\mathrm{R}} & \text { angular speed of the rotor } \\ \Omega_{\mathrm{F}} & \text { excitation circular frequency } \\ \overline{\hat{W}}^{\mathrm{U}} & \text { unbalance response amplitude } \\ \overline{\hat{\bar{F}}}^{\mathrm{U}} & \text { unbalance force amplitude } \\ \overline{\bar{\varepsilon}} & \text { unbalance parameter vector }\end{array}$

$\mathbf{M}, \mathbf{D}, \mathbf{G}, \mathbf{K}$

$\overline{\mathbf{H}}$

$\bar{\Phi}$

$\bar{\Lambda}$

\section{References}

Belz, J. (1997) Modalanalysegestützte Unwuchtidentifikation elastischer Rotoren, Schwingungen in rotierenden Maschinen IV, SIRM '97, Braunschweig, Wiesbaden, Vieweg-Verlag. modal matrix

spectral matrix
Ebersbach, P. (1989) Rechnerunterstützte Methoden der Messwerterfassung und Parameteridentifikation (Modalanalyse) und ihre Anwendbarkeit auf rotierende Maschinen. Dissertation, Institut für Mechanik, Universität-Gh Kassel.

Goodman, T.P. (1964) A least-squares method for computing balance corrections. Journal of Engineering for Industry 86, ASME, pp. 273-279.

Kellenberger, W. (1987) Elastisches Wuchten. Modale Verfahren, EK-Technik, Sondertechniken automatisches und thermisches Wuchten. Berlin, Heidelberg, New York, Springer-Verlag.

Krämer, E. (1984) Maschinendynamik. Berlin, Heidelberg, New York, Tokyo, Springer-Verlag.

Kreuzinger-Janik, T. (1995) Entwicklung eines Rotorprüfstandes unter Verwendung experimenteller und rechnerischer Modalanalyse. Institut für Mechanik, Diplomarbeit II, UniversitätGh Kassel.

Lee, C.-W. (1993) Vibration Analysis of Rotors. Dordrecht, Boston, London, Kluwer Academic Publishers. 


\section{ait \\ ENERGY MATERIALS}

M A N E Y publishing

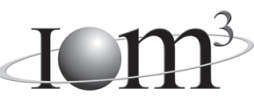

\section{Materials Science \& Engineering for Energy Systems}

Maney Publishing on behalf of the Institute of Materials, Minerals and Mining

The Institute of Materials, Minerals \& Mining

Economic and environmental factors are creating ever greater pressures for the efficient generation, transmission and use of energy. Materials developments are crucial to progress in all these areas: to innovation in design; to extending lifetime and maintenance intervals; and to successful operation in more demanding environments. Drawing together the broad community with interests in these areas, Energy Materials addresses materials needs in future energy generation, transmission, utilisation, conservation and storage. The journal covers thermal generation and gas turbines; renewable power (wind, wave, tidal, hydro, solar and geothermal); fuel cells (low and high temperature); materials issues relevant to biomass and biotechnology; nuclear power generation (fission and fusion); hydrogen generation and storage in the context of the 'hydrogen economy'; and the transmission and storage of the energy produced.

As well as publishing high-quality peer-reviewed research, Energy Materials promotes discussion of issues common to all sectors, through commissioned reviews and commentaries. The journal includes coverage of energy economics and policy, and broader social issues, since the political and legislative context influence research and investment decisions.

\section{CALL FOR PAPERS}

Contributions to the journal should be submitted online at http://ema.edmgr.com

To view the Notes for Contributors please visit: www.maney.co.uk/journals/notes/ema

Upon publication in 2006, this journal will be available via the Ingenta Connect journals service. To view free sample content online visit: www.ingentaconnect.com/content/maney

For further information please contact:

Maney Publishing UK

Tel: +44 (0)113 2497481 Fax: +44 (0)1132486983 Email: subscriptions@maney.co.uk

or

Maney Publishing North America

Tel (toll free): 8662975154 Fax: 6173546875 Email: maney@maneyusa.com

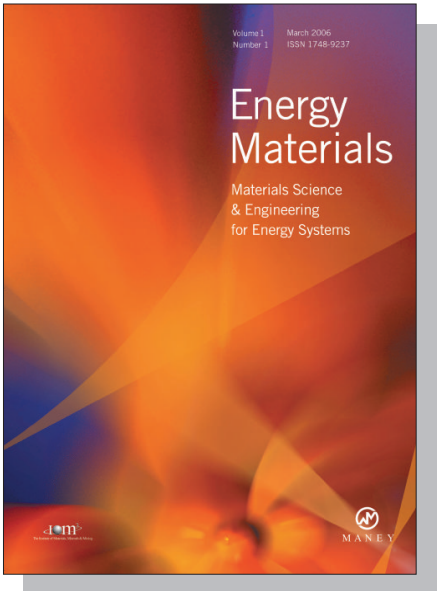

EDITORS

Dr Fujio Abe

NIMS, Japan

Dr John Hald, IPL-MPT, Technical University of Denmark, Denmark

Dr R Viswanathan, EPRI, USA

\section{SUBSCRIPTION INFORMATION}

Volume 1 (2006), 4 issues per year

Print ISSN: 1748-9237 Online ISSN: 1748-9245

Individual rate: $£ 76.00 / U S \$ 141.00$

Institutional rate: $£ 235.00 /$ US $\$ 435.00$

Online-only institutional rate: $£ 199.00 / U S \$ 367.00$

For special $\mathrm{IOM}^{3}$ member rates please email

subscriptions@maney.co.uk

\section{For further information or to subscribe online please visit www.maney.co.uk}



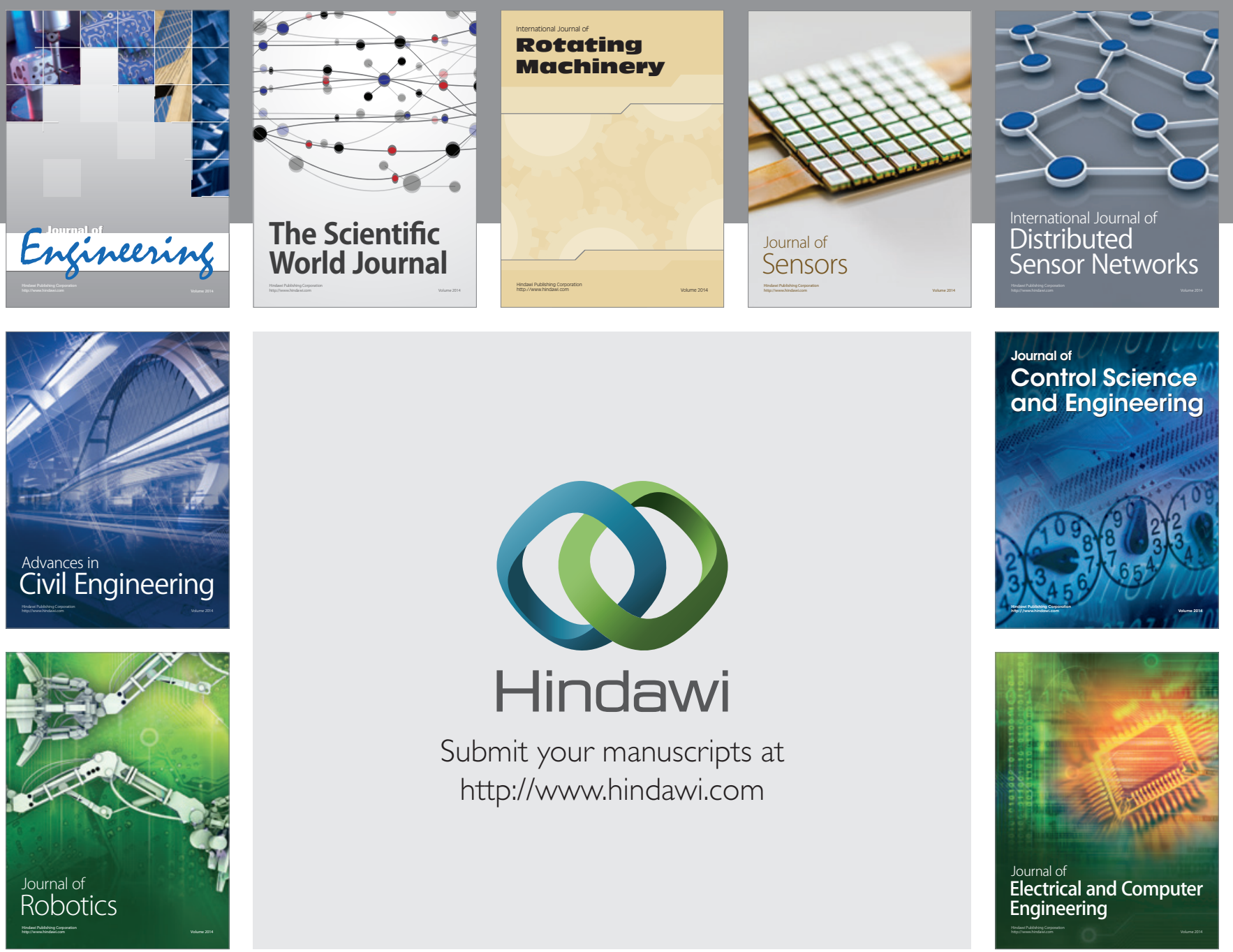

Submit your manuscripts at

http://www.hindawi.com
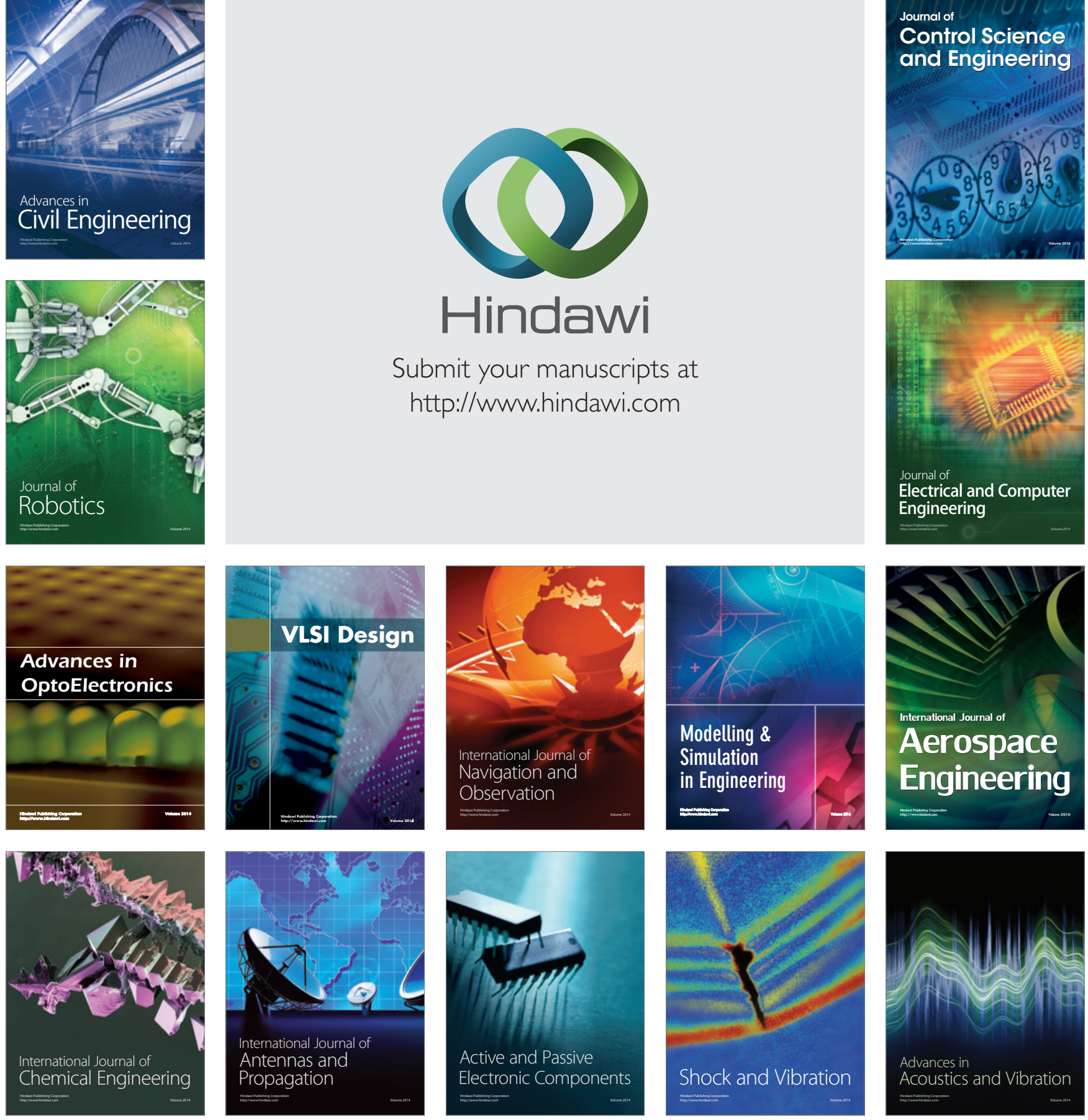